\title{
Downlink Spectrum Allocation in 5G HetNets
}

\author{
Tamer R. Omar, Ahmed E. Kamal, J. Morris Chang \\ Department of Electrical and Computer Engineering, Iowa State University \\ \{tomar, kamal, morris\}@iastate.edu \\ (Invited Paper)
}

\begin{abstract}
Fifth generation mobile systems (5G) target an Average Area Spectral Efficiency (AASE) over hundred Gbps $/ \mathrm{km}^{2} /$ user for future mobile systems with an Energy Dissipation (ED) per unit area similar to the current ED levels. Heterogeneous networks (HetNets) with high density of deployed small cells are currently adopted to aid in achieving the target ED and AASE by 5G. Limited spectrum availability requires efforts to manage the spectrum utilization in such dense deployments. Development of new network architectures and Radio Resources Management (RRM) schemes is important to address such challenges. The objective of this work is to propose a new architecture that consists of a Decision Support System (DSS) and a data collection system to dynamically manage and control the spectrum allocation process. The DSS generates spectrum allocation patterns using non-parametric estimation and statistical analysis for the collected data. A new RRM model using a Plan, Do, Control and Act (PDCA) cycle is proposed as a new self optimization module in the self organizing network framework. The PDCA model utilizes the new architecture and the allocation patterns to dynamically predict future spectrum allocation. Results show improvement in the AASE achieved using the PDCA model compared to conventional spectrum allocation.
\end{abstract}

Index Terms-5G, HetNets, Dynamic Spectrum Management, AASE.

\section{INTRODUCTION}

$\mathrm{T}$ He Cisco visual networking index forecasts that the dependence on mobile phone and data services by mobile subscribers will increase 10-15 fold between 2012 and 2017 [1]. Major mobile service providers (MSPs), standardization bodies and different forums are exerting maximum efforts to find novel solutions that offer users high quality services which meet their expectations. The mobile and wireless communications enablers for the twenty-twenty information society (METIS) is leading the first international and largescale research activity on 5G [2]. Efforts on 5G are supposed to produce a new standard that will replace the current $4 \mathrm{G}$ technologies into the next decade.

Coordinated multi-point (COMP) transmission/reception, carrier aggregation, MSPs network (e.g. spectrum) sharing, advanced multiple-input multiple-output techniques, relays and enhanced inter-cell interference coordination are the enabling technologies for $4 \mathrm{G}$ and act as the baseline for $5 \mathrm{G}$. The authors in [3] provide an overview for these enabling technologies from the physical layer aspects while [4] explains the possible improvements and the associated challenges for those enabling technologies.

978-1-4799-0959-9/14/\$31.00 @2014 IEEE
Rate adaptive, margin adaptive, and utility based dynamic spectrum allocation (DSAn) and physical resources allocation (PRAn) schemes are suggested in previous work to improve spectrum utilization [5]. However, Heterogeneous Networks (HetNets) which consist of conventional macro cells overlaid by small cells are currently proposed as the most feasible method to improve spectrum utilization specially that spectral efficiency has reached its limit. DSAn and PRAn are implemented using rate adaptive and COMP schemes in [6], [7] to maximize the system throughput. Geometric programming is used in [6] to optimize users minimum rate under variable bandwidth and variable power. Resource partitioning is proposed in [7] to improve the performance of HetNets using a two cell selection criterion, one based on maximizing the received signal strength with a potential bias and the other based on maximizing the product of signal to interference plus noise ratio (SINR) and bias.

Self organizing networks (SON) for HetNets are discussed in [8] with a main goal of reducing capital and operational expenditure that increase due to the increased number of network parameters to be monitored. Also other different autonomic functionalities like auto configuration, self optimization, diagnosis, and self healing are evaluated.

Traffic Geo-Location service is the service of identifying the real-time geographic location and the traffic load of user equipments (UEs). The benefits and technical issues of traffic Geo-Location service is explored in [9]. Geo-Location service is discussed as a tool that aid MSPs to deliver an optimized environment to deploy macro cells (MCs) to small cells (SCs) off loading solutions.

According to the best of our knowledge none of the previous approaches presents a module in the SON framework to operate as a decision support system (DSS) that helps automate the optimization process of the PRAn and DSAn. Thus, this paper develops a new architecture and a supporting SON module to optimally distribute and utilize the available resources and frequency spectrum in order to maximize the benefit of the available limited spectrum. A traffic Geo-location resources allocation database (Geo-LRADB) is proposed to be populated using Geo-Location service to store the geographical information of the users allocated resource blocks (RBs).

The goal of this paper is to address the DSAn and PRAn problem using a new allocation approach

1) A new four step Radio Resource Management (RRM) model, referred to as the PDCA (Plan-Do-Check-Act), is proposed. The model aims at identifying the optimal DSAn and PRAn in terms of MSPs return on investment 


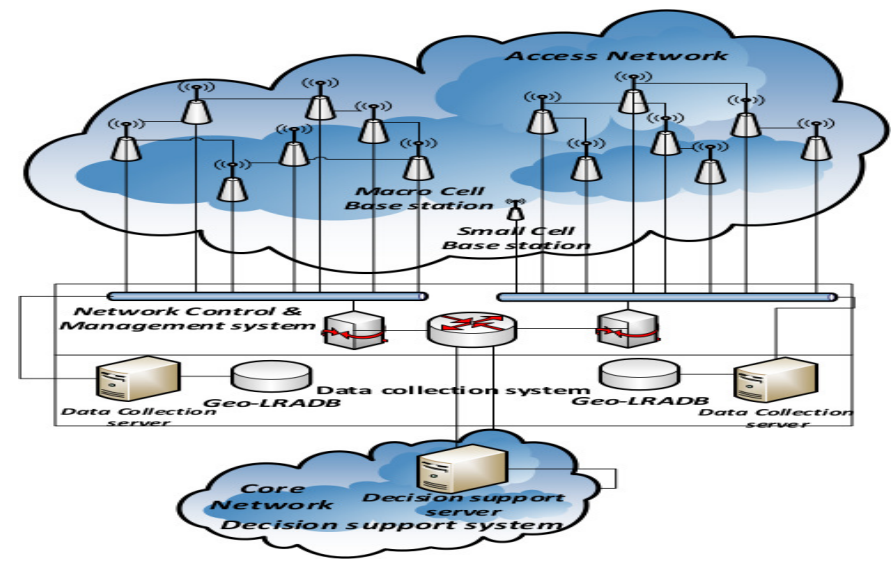

Fig. 1: Network architecture

(ROI) by enhancing the average area spectral efficiency in HetNets.

2) A new network architecture is proposed that consists of two components

- A data collection system (DCS) infrastructure that includes a Geo-LRADB is introduced to the distributed network control and management system (NCMS) in the network architecture whose purpose is to aid as a traffic data repository.

- A DSS that applies the PDCA model is introduced as a new self optimization module within the SON framework. The model represents the core functionality of the DSS and utilizes the data collected by the distributed NCMS to predict and recommend the optimal DSAn to be implemented in the network.

3) A non-parametric estimation analysis and a stochastic linear programming with recourse is formulated and adopted to enhance the spectrum allocation by utilizing the previous history for predicting future allocations.

The remainder of this paper is organized as follows. Network and system model is introduced in Section II. Section III provides details about the RRM mechanism in HetNets. The RRM module proposed for DSAn self-optimization in the SON framework is presented and the optimization problem is formulated in Section IV. Simulation results are presented and discussed in Section V. Finally, in Section VI conclusions are drawn.

\section{NETWORK AND SYSTEM MODEL}

This section introduces the proposed HetNet architecture, system model, and the power allocation model.

\section{A. Network Architecture}

The proposed architecture adds a DSS and a DCS to the current network architecture as shown in Fig. 1.

- A core network $(\mathrm{CN})$ central DSS consists of a decision support server (DSSrvr) and DSAn optimization methodology is proposed. The DSS is recommended for implementation as a radio resources self optimization module in the SON frame work. The DSS is responsible for globally planning and controlling the DSAn in the access network (AN).

- A DCS contains a group of data collection servers (DCSrvrs) and a Geo-LRADBs replicated in the NCMS and distributed over the AN to collect all necessary data required by the radio resources allocation process.

The data collected by the DCS is analyzed by the DSS to centrally manage the dynamic spectrum allocation self optimization in the HetNet, as described below :

1) Decision Support System: The DSS is a central management system using a DSSrvr to perform the needed functions. The DSS, in addition to the dynamic control and management of the DSAn self optimization, can be used in optimally controlling and managing multiple parameters in the AN, and recommending deployment plans and strategies to the NCMS. Examples of the tasks that can be managed by the DSS are ;

- Dynamically controlling the SINR threshold between different frequency partitions (FPs).

- Updating COMP deployment plan for Macro Cell-Base Stations (MC-BSs) and the deployment plan of the small cell-base stations (SC-BSs) inside the MCs.

- Dynamically changing the MC-BS beam-forming strategies.

- Managing the adaptive frequency reuse power patterns,

- Performing resource allocation trending analysis.

2) Data collection System: The DCSrvrs and a Geo-LRDBs proposed in the DCS within the NCMS perform several tasks;

- Data collection: the real time physical resource allocation in the AN is collected.

- Data aging: the old data that is no longer needed is aged and deleted according to the aging rules.

- System maintenance: the Geo-LRADB relational database management system is maintained.

\section{B. System Model}

We consider the down-link transmission in a multi-cell HetNet. Fig. 2 shows an example network which consists of seven identical adjacent hexagonal MC-BSs. Five external SC-BSs $(E S C s)$ are deployed outside of the AN coverage to extend coverage. Three internal SC-BSs $(I S C s)$ are used inside of the AN coverage for capacity increase. The maximum number of FPs in each MC in the AN is $M$ and each FP is denoted by $\mathcal{F}_{m c, i}$, where $i=\{1,2, \ldots, M\}$.

\section{Power Allocation Model}

1) Power Patterns: The idea of using power patterns is introduced by WiMAX [10] and can be extended to $5 \mathrm{G}$ networks to aid as a transmission power adaptation technique. Power patterns are used to specify the maximum allowed transmission power level in each FP. Different patterns have different maximum power levels allocated according to the area served by the FP (cell center FP, cell edge FP or SC FP). Fig. 3 shows the different power patterns used by the MC$\mathrm{BSs}$, the max power level used in each FP in the MC and the power patterns used by the SC-BSs for our system model that partition the utilized spectrum using reuse-3 partitioning as 


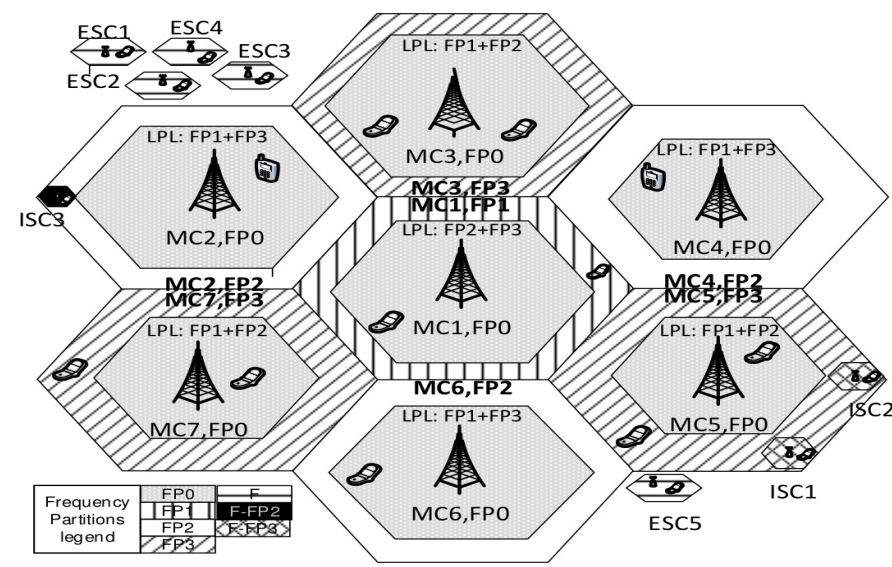

Fig. 2: System model

shown in Fig. 2: For example $M C_{1}$ uses pattern 1 where $P_{0}$ is used in the cell center for FP $\mathcal{F}_{0}$, and Low Power Level (LPL) $P_{2}$ is used for FP $\left(\mathcal{F}_{2}, \mathcal{F}_{3}\right)$ to avoid Inter-Cell Interference (ICI). $P_{1}$ is used in the cell edge for FP $\mathcal{F}_{1}$ to enhance the spectrum utilization by improving the UEs transmission rates. $M C_{2,4,6}$ use pattern 2, and $M C_{3,5,7}$ use pattern 3 to specify the maximum power levels in different FPs. $E S C_{1,2,3,4,5}$ use pattern $4, I S C_{1,2}$ implemented in $M C_{5}$ edge use pattern 5 where UEs use LPL $P_{2}$ in all the spectrum $\mathcal{F}$ except in FP $\mathcal{F}_{3}$ to avoid interference with UEs in $M C_{5}$ edge. $I S C_{3}$ use pattern 6 to specify the maximum power level in the SCs.

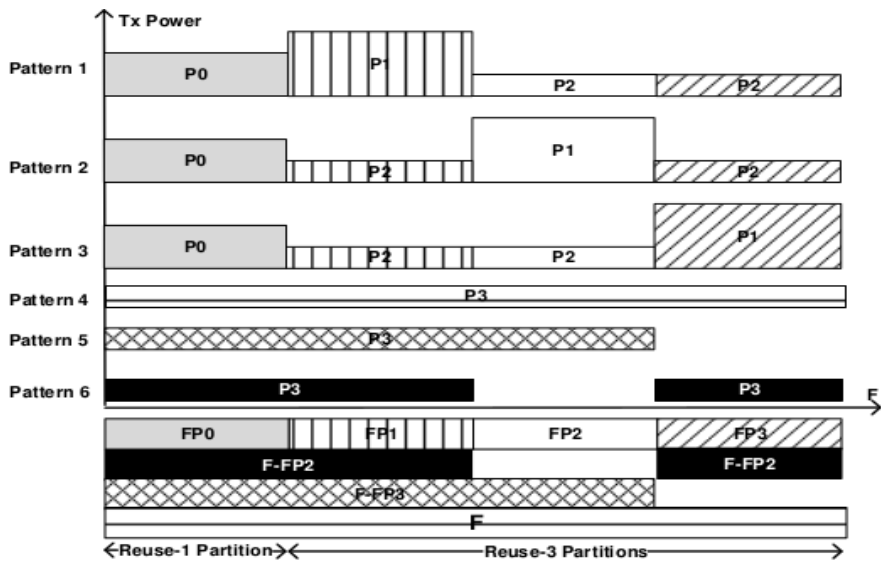

Fig. 3: Power Patterns

2) Power Levels: Fig. 3 maps the adaptive power allocation used by MC-BSs in the different FPs and the SC-BSs of the system model shown in Fig. 2. FP $\mathcal{F}_{0}$ is reused in the center of each MC with a fixed maximum power level $P_{0, \max }$. FPs $\left(\mathcal{F}_{1}, \mathcal{F}_{2}, \mathcal{F}_{3}\right)$ are reused with a maximum power boosted level $P_{1, \max }$ in the MC edges and a maximum LPL $P_{2, \max }$ in the cell center. All spectrum $\mathcal{F}$ is reused in $E S C_{1,2,3,4,5}$ with a maximum LPL $P_{3, \text { max }}$. FP $\left(\mathcal{F}-\mathcal{F}_{3}\right)$ is reused in SCs $I S C_{1,2}$ and $\mathrm{FP}\left(\mathcal{F}-\mathcal{F}_{2}\right)$ is used in SC $I S C_{3}$ with a LPL $P_{3, \max }$.

The utilized Adaptive Fractional Frequency Reuse (AFFR) mechanism decides the maximum power levels and power patterns to be used in the AN. Adaptive power allocation improves the energy dissipation by adapting the UE transmission power levels according to their locations. Thus, the MC-BS and SC-BS use specific power patterns to aid in mitigating
ICI between different FPs in the AN.

\section{Radio Resource Management}

DSAn and PRAn are two extremely important RRM functions that allocate the amount of spectrum to each FP and the amount of RBs to each UE, respectively. This section introduces the down-link frequency partition configuration (DFPC) basics, frequency allocation hierarchy in a HetNet, the DSAn utility based optimization problem formulation and the Geo-LRADB structure.

\section{A. Down-link Frequency Partition Configuration}

AFFR is a promising mechanism adopted by $4 \mathrm{G}$ technology to enhance the spectral efficiency in the AN by controlling the frequency partition size (FPS), frequency partition count (FPCT), and UEs maximum transmission power limits.

The maximum number of utilized DFPCs in the AN is $\mathbb{C}$ and each DFPC is denoted by $C_{c}$, where $c \epsilon\{1,2, \ldots, \mathbb{C}\}$. The maximum number of utilized DFPCs is determined according to the utilized bandwidth $(\mathcal{W})$ and the employed Fast Fourier Transform (FFT) in the AN.

A list of the DFPCs that can be dynamically implemented in the AN according to the user requirements and the network load conditions are presented in [10]. Each DFPC specifies the number of FPs in terms of their FPS and FPCT.

\section{B. Dynamic Spectrum Allocation}

DSAn is calculated centrally by the DSS and implemented globally in the AN to control the distribution of the RBs between MC-BSs cell regions and SC-BSs existing in a HetNet. DSAn aims at performing the optimal spectrum allocation by identifying the best DFPC to be used in the AN.

1) Distributed Utility Formulation: A number of utility based frameworks for scheduling RBs to UEs in the AN are proposed in previous work. Allocation schemes are presented to calculate the allocation matrices of scheduling the RB $k$ to UE $j$ for each FP $i$ in MC-BS $b$ in order to optimize a utility function $U_{i j k}$. The distributed maximum utility denoted by $U_{b, \max }$ is calculated by maximizing the summation of the aforementioned utility $\left(\max \sum_{i} \sum_{j} \sum_{k} U_{i j k} x_{i j k}\right)$ for all UEs in each MC.

2) Central Utility Formulation: The proposed central utility function is used to identify the optimal DFPC. The central utility function is denoted by $U_{c, \max }$ and is calculated globally by the DSS. $U_{c, \max }$ is the sum of $U_{b, \max }$ achieved by all MCBSs and SC-BSs in the AN. The DSS calculates $U_{c, \max }$ per configuration $C_{c}$ as $U_{c, \max }=\sum_{b=1}^{B} U_{b, \max }$ and uses it in identifying the DFPC to be implemented in the AN.

3) DSAn Problem Formulation: The DCS stores the real time allocation parameters used to achieve $U_{b, \max }$ in the AN at the Geo-LRADB. The expected central utility for all DFPCs are calculated by the DSS periodically each time interval $(T)$ using $U_{b, \max }$, where $T$ is the DFPC update interval. The maximum central utility $U_{c, \max \text {,Actual }}$ that is achieved due to the implementation of a specific DFPC in the AN during a $T$ interval is denoted as $U_{D S S}$. The calculation of 
$U_{c, \text { max }, \text { Calculated }}$ for all DFPCs requires access to a significant amount of information. One way to reduce the amount of information is by limiting the calculations of $U_{c, \max , \text { Calculated }}$ to only the DFPCs that can possibly be implemented in the AN. An example of the possible DFPCs is the group of DFPCs with a same FPCT.

The DCS calculates the UEs' expected achievable data rates $r_{i j k, \text { calculated }}$ for all DFPCs. The UEs' expected SINR values $S I N R_{i j k, \text { calculated }}$ for all DFPCs are calculated using the reported values, $S I N R_{i j k, A c t u a l}$, by the UEs. In order to calculate the UEs expected utility $U_{i j k, \text { calculated, DCS }}$ calculates the UEs expected normalized spectral efficiency $N S E_{i j k, \text { calculated }}$ as

$$
N S E_{i j k}=\frac{r_{i j k}(1-P E R)}{R M(i) * \mathcal{W}_{i}}
$$

where PER and $R M(i)$ are the packet error rate and the resource metric, respectively. The DCS also calculates the expected power consumption $P_{i j k, \text { calculated }}$ is calculated for all DFPCs as

$$
P_{i j k}=A * \frac{2^{r_{i j k} / \mathcal{W}_{j}}}{\rho * S I N R_{i j k}}
$$

where $A$ is the area calculated from the Geo-location information collected in the Geo-LRADB, $\rho=\frac{-1.5}{\ln (5 * B E R)}$ is constant and BER is bit error rate. After calculating the expected utility $U_{i j k, \text { calculated }}$ as

$$
U_{i j k, \text { calculated }}=\frac{N S E_{i j k, \text { calculated }}}{P_{i j k, \text { calculated }}}
$$

the DCS calculates the expected maximum distributed utility $U_{b, \text { max, calculated }}$ for all current group DFPCs.

The optimal DFPC is identified by aggregating $U_{b, \text { max,calculated }}$ for all MC-BSs and SC-BSs per DPFC and comparing them to choose the DFPC with $U_{c, \text { max,calculated }}$. The DSS aided by the information collected in the DCS calculates the $U_{c, \max , \text { calculated }}$ achieved during the most recent $T$ interval by solving the problem below:

$$
\begin{array}{cc}
\max & \sum_{c=1}^{C} U_{c, \max , \text { Calculated }} x_{c} \\
\text { s.t } & \sum_{c=1}^{\mathbb{C}} x_{c}=1 \\
& x_{c}=\{0,1\}
\end{array}
$$

where $X_{c}$ is the assignment vector and the binary variable $x_{c} \in X_{c}$ is the assignment indicator. $x_{c}=1$, if $C_{c}$ is the assigned DFPC, and $x_{c}=0$ otherwise. In this trivial optimization problem, every $T$ interval the DSS compares the $U_{c, \text { max }, \text { Calculated }}$ for all DFPCs to select the DFPC with the maximum $U_{c, \text { max }, \text { calculated }}$ for implementation in the next $T$ interval.

\section{Geo-Location Resource Allocation Database}

The Geo-LRADB is a relational database that we proposed in this paper as a repository for the data needed in the optimization of frequency spectrum and the identification of the DFPC by the DSS.

A relational database model is updated in the DCS to collect the RRM data from the MC-BS. The collected data is used to calculate the expected distributed and central utilities in future $T$ intervals.

\section{Radio Resource Management in SElF ORGANIZING NETWORKS}

A new RRM model is proposed in this section to act as the core methodology used by the DSS for managing and controlling the radio resources in the network. The new RRM model denoted as the PDCA model is suggested for continuous improvement of the spectrum dynamic allocation. The DSS is recommended to be added as a self optimization module in the SON framework to automate the dynamic spectrum allocation process. A spectrum allocation methodology that relies on the statistical analysis of the collected data and a problem formulation using stochastic programming with recourse is proposed.

\section{A. Plan-Do-Check-Act Model}

The PDCA model virtually calculates the DSAn and PRAn allocation for all $C_{c}$ to recommend the DFPC changes in the AN that achieve $U_{c, \max }$. The PDCA model learning cycle has four repeatable steps; 1) Plan, 2) Do, 3) Check, and 4) Act. The functions of each of the four steps are:

- Plan: Understand the gap between the real time central utility $U_{c, \max \text {, Actual }}$ calculated due to the current DFPC and the expected $U_{c, \max , \text { Calculated }}$ calculated for other DFPCs.

- Do: Using the SON resources allocation self optimization module, implement the optimal DFPC to be used in the AN according to the data collected in the Act phase.

- Check: Observe the change on the achieved distributed utility $U_{b, \max , \text { Actual }}$ after applying the updated DFPC .

- Act: Collect the new data by the DCS in the Geo-LRADB and communicate it to the DSS.

\section{B. Dynamic Spectrum Allocation}

The analysis of the DSAn dynamics during the PDCA cycle is important to build allocation patterns which are used in a stochastic program to estimate the DFPC to be implemented in future intervals

1) Patterns Analysis : A transition probability matrix (stochastic matrix) is used to obtain allocation patterns from the data collected at Table $_{3}$ and Table $_{4}$ in the Geo-LRADB. The utilization of the stochastic matrix helps in building a DSAn pattern based on the empirical data collected in the DCSrvrs and analyzed by the DSS module implemented in the SON framework. This allocation pattern can be used for future application of the DFPC to be implemented in HetNets. 
Table I: Model variables

The continuous variations in the network load encountered due to changes in users' distributions and traffic requirements in each cell has an important effect on the DSAn process. The DFPC needs to change periodically every DFPC update interval $(T)$ in order to address variations in the achieved utility $U_{c, \text { max,Actual }}$ due to network load dynamics.

Using empirical data, we construct the time homogeneous transition probability matrix, $P^{T_{n-1}, T_{n}}$ of size $(\mathbb{C} x \mathbb{C})$, with elements $p_{i j}^{T_{n-1}, T_{n}}$ expressing the transition from DFPC $i$ at current allocation time $T_{n-1}$ to DFPC $j$ at future allocation time $T_{n}$ defined as

$p_{i j}^{T_{n-1}, T_{n}}=p\left\{U_{j}^{T_{n}}\right.$ is optimal at time $\mathrm{n} \mid U_{i}^{T_{n-1}}$ was optimal at time n-1)

for any $c_{i}$ which is the DFPC achieving the optimal central utility $U_{c, \max , \text { Actual }}$ at allocation time $T_{n-1}$ and $c_{j}$ which is the DFPC achieving the maximum central utility $U_{c, \text { max }, \text { Calculated }}$ at allocation time $T_{n}$. DFPC is always determined after finding the UEs locations from the Geolocation database. The transition probability matrix is recalculated every allocation time to consider the last transition in calculating the probability matrix. A non-parametric estimate (histogram model) is used to present the distribution of the empirical data utilized in calculating the probability distribution $p\left\{U_{j}^{T_{n}} \mid U_{i}^{T_{n-1}}\right)$.

2) Problem Formulation: A stochastic integer linear program with recourse is used to calculate the maximum util-

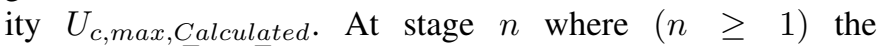
values of $p_{i j}^{T_{n-1}, T_{n}}$ of random variable $\xi$ in the stochastic matrix $P^{T_{n-1}, T_{n}}$ as well as the previous actual utilities $\left(U_{c, \text { max }, \text { Actual }}^{T_{0}}, \ldots, U_{c, \text { max }, \text { Actual }}^{T_{t}}, \ldots, U_{c, \text { max }, \text { Actual }}^{T_{n-1}}\right)$ can be calculated using the DSS historical data. The recourse in the stochastic program is used in order to represent the expected deficiency resulting in network utilization $U_{D S S}$ at time $T_{n}$ due to the choice of a a certain DFPC according to the calculated utility $U_{c, \max , \text { Calculated }}$. This penalty is enforced to account for the DSS learning from previous decisions in identifying the future DFPC recommendations. The penalty incurred is determined after the observation of the empirical data and calculating the possible difference in the calculated central utility $U_{c, \max , \text { Calculated }}$, which makes the $x_{c}$, a first stage decision variable.

The stochastic integer linear program with recourse costs at time $T_{n}$ is modeled as

$$
\begin{gathered}
\max \sum_{c=1}^{\mathbb{C}} U_{c, \text { max }, \text { Calculated }}^{T_{n}} x_{c}^{T_{n}}- \\
\sum_{j=1}^{\mathbb{C}} \sum_{c=1}^{\mathbb{C}} P_{i j}^{T_{n-1}, T_{n}}\left[y_{j}\right]^{+} x_{c}^{T_{n}} \\
\sum_{c=1}^{\mathbb{C}} x_{c}^{T_{n}}=1 \\
x_{c}^{T_{n}}=\{0,1\}
\end{gathered}
$$

equation (8) evaluates the penalty $\left[y_{j}\right]^{+}$as

\begin{tabular}{|c|c|}
\hline$T_{n}$ & Allocation Time \\
\hline$U_{c, \text { max }, \text { calc }}$ & Max utility calculated by the DSS \\
\hline$U_{c, \max , \text { Act }}$ & Actual utility collected by the DCS \\
\hline$p_{i j}^{T_{n-1}, T_{n}}$ & Transition probability matrix \\
\hline$\left[y_{j}\right]^{+}$ & Penalty due to previous allocation \\
\hline$x_{c}^{T_{n}}$ & Allocation Matrix \\
\hline
\end{tabular}

$\left[y_{j}\right]^{+} s, t \quad \begin{cases}y_{j}=\left(U_{j, \max , \text { Calc }}^{T_{n-1}}-U_{c, \text { max }, \text { Act }}^{T_{n-1}}\right) & \forall y_{j} \geqslant 0 \\ y_{j}=0 & \text { otherwise }\end{cases}$

Table I shows the key variables of the model. The solution of (5) to (7) identifies the DFPC that achieves the expected maximum utility in the AN in the next $T$ interval.

\section{Simulation and Results}

This section presents a comparison between the two DSAn schemes proposed, first scheme using the equations (1)-(3) of section III and second scheme using (5)-(7) of section IV. We used this schemes to calculate $U_{c, \max , \text { Calculated }}$ and identify the optimal DFPC in the AN.

The first scheme is a memory-less scheme using integer linear programing and the second scheme is memory aided using stochastic programming with recourse to identify the optimal DFPC to be implemented in the AN and the schemes are denoted by $S_{1}$ and $S_{2}$ respectively. Simulation for DFPCs with four FPs $\left(C_{3}\right.$ to $\left.C_{7}\right)$ is conducted and results are presented using simulation parameters in Table II.

Table II: System parameters

\begin{tabular}{|c|c|}
\hline Parameter & Value \\
\hline Number of Cells $(B)$ & 7(MC) - 5(ESC) - 3(ISC) \\
\hline Number of FPs $(M)$ & 4 \\
\hline Number of UEs in MC & 60 \\
\hline Number of UEs in each SC & 20 \\
\hline Number of RBs & 96 \\
\hline Number of DFPC update interval $(T)$ & 360 \\
\hline Number of DFPCs $(c)$ & $5\left(C_{3}-C_{7}\right)$ \\
\hline MC-BS Maximum Power $\left(P_{\max }\right)[w]$ & 25 \\
\hline SINR Threshold $\left(\delta_{b}\right)[d b]$ & 18.5 \\
\hline Bandwidth $\left(\mathcal{W}_{i}\right)[M H Z]$ & 20 \\
\hline
\end{tabular}

A comparison between the DFPC allocated by scheme $S_{1}$ and scheme $S_{2}$ is presented in Fig. 4. The results show that $S_{1}$ and $S_{2}$ have different allocation decisions, the decisions taken by $S_{2}$ are affected by the history of the previous allocations collected in the DCSrvrs.

The network operation is simulated by conducting two consecutive simulation runs. In the first run the DFPC transition probability matrix, $P^{T_{n-1}, T_{n}}$ is created using the normal allocation model $S_{1}$ for a sufficient number of iterations to capture the UEs dynamics in the network. In the second run the DFPC transition probability matrix is used in the stochastic allocation model $S_{2}$ to calculate the penalty incurred due to previous allocations. The expected DFPCs are then calculated by solving the optimization problems for both models. The expected DFPCs recommended by the DSS are used to operate the network and the central utility $U_{c, \text { max,actual }}$ is measured each DFPC update interval to evaluate the network utilization. 


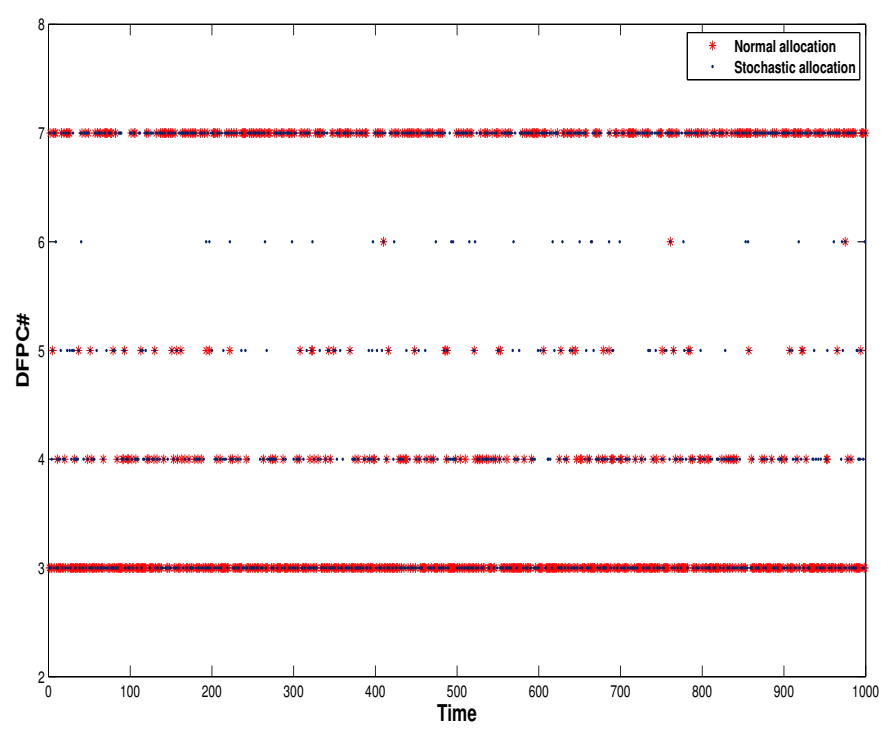

Fig. 4: DFPC: Comparison between $S 1$ and $S 2$.

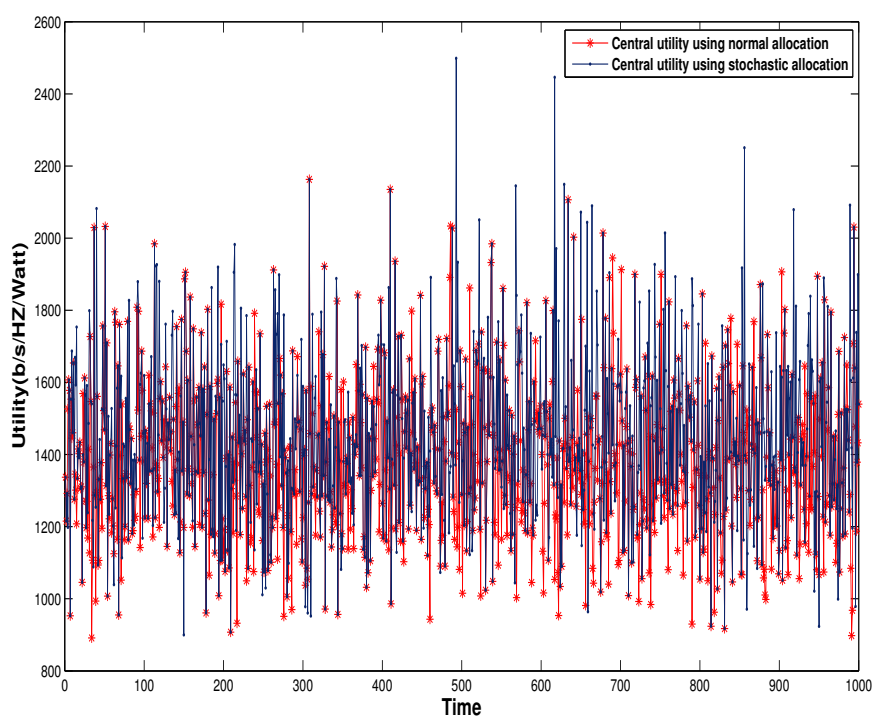

Fig. 5: $U_{c, \max }$ : Comparison between $S 1$ and $S 2$.

The results in Fig. 5 show a comparison between the measured maximum central utility $U_{c, \text { max,actual }}$ for $S_{1}$ and $S_{2}$. Results show that the allocation of the DFPC using $S_{2}$ is achieving better central utility $U_{c, \text { max,actual }}$ than that achieved according to $S_{1}$. The allocation of the DFPC using $S_{1}$ outperforms $10.3 \%$ of the time while $S_{2}$ outperforms 33.2 $\%$ and both models generate the same DFPC allocation 56.5\% of the time. Thus the results verified that $S_{2}$ outperforms $S_{1}$ for $22.8 \%$ of the time. Also the average central utility $U_{c, \text { max }, \text { Actual }}$ using $S_{2}$ is calculated as $1536.5 \mathrm{~b} / \mathrm{s} / \mathrm{Hz} /$ watt while for $S_{1}$ it sums up to $1416.1 \mathrm{~b} / \mathrm{s} / \mathrm{Hz} /$ watt with a significant difference of $120.4 \mathrm{~b} / \mathrm{s} / \mathrm{Hz} /$ watt between the two allocation models..

\section{CONCLUSION}

The mobile and wireless communications enablers for the twenty-twenty information Society (METIS) in their research activities on 5G systems are targeting an average area spectral efficiency (AASE) over hundred $\mathrm{Gbps} / \mathrm{km}^{2} /$ user. Heterogeneous networks (HetNets) that consist of both macro and small cells are currently viewed as the most capable solution to satisfy the expected future AASE requirements.

This work presents a new network architecture for the next generation $(5 \mathrm{G})$ of wireless communication systems. The architecture aims at building a self organizing network (SON) module in the core network. The module acts as a decision support system (DSS) supported by a Geo-location data collection infrastructure for radio resources utilization. The new network architecture using the DSS is expected to manage the spectrum utilization by forecasting the best downlink frequency partition configuration (DFPC) to be utilized in the access network (AN).

Finally a plan-do-control-act (PDCA) model for radio resource management is proposed to be used by the DSS in the SON module. The PDCA model aims at continuous improvement of the spectrum allocation process. A statistical analysis for the empirical data collected in a proposed data collection system (DCS) is performed by the DSS. Utilizing the DSS and the collected data, an optimization problem using stochastic linear programing with recourse and a discrete probability distribution modeled as a histogram is formulated to identify the best DFPC to be implemented in the AN. Simulations is conducted and results for the proposed scheme show that identifying future DFPC by learning from old allocations and using stochastic allocation with recourse improves the spectrum utilization efficiency over using a memoryless allocation method.

\section{REFERENCES}

[1] "Cisco Visual Networking Index: Global Mobile Data Traffic Forecast Update, 2012-2017," white paper, Cisco, Febr. 2013."

[2] Mobile and wireless communications Enablers for the Twenty-twenty Information Society (METIS), Intermediate description of the spectrum needs and usage principles, Deliverable D5.1, , 2013.

[3] T.-T. Tran, Y. Shin, and O.-S. Shin, "Overview of enabling technologies for 3GPP LTE-advanced," EURASIP Journal on Wireless Communications and Networking, 2012.

[4] I. F. Akyildiz, D. M. Gutierrez-Estevez, and E. C. Reyes, "The evolution to 4G cellular systems: LTE-Advanced," Physical Communication, vol. 3, no. 4, pp. $217-244,2010$.

[5] D. Ling, Z. Lu, Y. Ju, X. Wen, and W. Zheng, "A multi-cell adaptive resource allocation scheme based on potential game for ICIC in LTE-A," International Journal Communication Systems, 2013.

[6] B. Krasniqi and C. Mecklenbrauker, "Maximization of the Minimum Rate by Geometric Programming for Multiple Users in Partial Frequency Reuse Cellular Networks," in IEEE Vehicular Technology Conference (VTC Fall), 2011, pp. 1-5.

[7] K. Balachandran, J. Kang, K. Karakayali, and K. Rege, "Cell Selection with Downlink Resource Partitioning in Heterogeneous Networks," in IEEE International Conference on Communications Workshops (ICC), 2011, pp. 1-6.

[8] O. Østerbø and O. Grøndalen, "Benefits of Self-Organizing Networks (SON) for Mobile Operators," Journal of Computer Networks and Communications, vol. 2012, no. 862527, p. 16, 2012.

[9] O. Guéret, "Exploring Traffic Geo-Location as a Key Strategy to Offload Macro Networks with Small Cells Solutions," Alcatel-Lucent, 2011, http://webform.alcatel-lucent.com/res/alu/survey/alu2CustomForm.jsp? $\mathrm{cw}=$ alu2CorpDocDownload\&LMSG_CABINET=Docs_and_Resource_ Ctr\&LMSG_CONTENT_FILE=White_Papers/Alcatel-Lucent_GeoLocation.pdf.

[10] IEEE, IEEE Standard for Local and metropolitan area networks Part 16: Air Interface for Fixed and Mobile Broadband Wireless Access Systems, May 2009. 